\title{
Mario Kozah, Abdulrahim Abu-Husayn, Saif Shaheen Al-Murikhi, Haya Al Thani (eds). An Anthology of Syriac Writers from Qatar in the Seventh Century
}

Florence Jullien

\section{(2) OpenEdition}

\section{Journals}

Édition électronique

URL : http://journals.openedition.org/abstractairanica/45203

DOI : 10.4000/abstractairanica.45203

ISBN : 1961-960X

ISSN : 1961-960X

Éditeur :

CNRS (UMR 7528 Mondes iraniens et indiens), Éditions de l'IFRI

\section{Référence électronique}

Florence Jullien, « Mario Kozah, Abdulrahim Abu-Husayn, Saif Shaheen Al-Murikhi, Haya Al Thani (eds). An Anthology of Syriac Writers from Qatar in the Seventh Century ", Abstracta Iranica [En ligne], Volume 37-38-39 | 2018, document 36, mis en ligne le 30 décembre 2018, consulté le 28 septembre 2020. URL : http://journals.openedition.org/abstractairanica/45203 ; DOI : https://doi.org/10.4000/ abstractairanica.45203

Ce document a été généré automatiquement le 28 septembre 2020.

Tous droits réservés 


\title{
Mario Kozah, Abdulrahim Abu- Husayn, Saif Shaheen Al-Murikhi, Haya Al Thani (eds). An Anthology of Syriac Writers from Qatar in the Seventh Century
}

\author{
Florence Jullien
}

\section{RÉFÉRENCE}

Mario Kozah, Abdulrahim Abu-Husayn, Saif Shaheen Al-Murikhi, Haya Al Thani (eds). An Anthology of Syriac Writers from Qatar in the Seventh Century. Piscataway: Gorgias Press, 2015, 736 p. ISBN 978-1-4632-0545-4, (Gorgias Eastern Christian Studies 39)

1 Cette anthologie fait suite à un colloque tenu à Qatar University (Doha) en partenariat avec l'Université américaine de Beyrouth en 2014, dans le cadre d'un programme de recherche financé par le Qatar National Research Fund. Elle rassemble des écrits monastiques et ascétiques rédigés par des auteurs syriaques des $\mathrm{VII}^{\mathrm{e}}$ et $\mathrm{VIII}^{\mathrm{e}}$ siècles originaires de la province ecclésiastique du Bēth-Qațrāyē (le Qatar actuel), dans des domaines aussi variés que l'hagiographie, la littérature épistolaire, exégétique, liturgique, canonique, spirituelle, et ascétique. Des extraits des principales œuvres sont traduits en anglais (certains pour la première fois), mis en contexte et annotés.

2 L'ouvrage s'ouvre avec l'Histoire de Mār Yonān attribuée à Mār Za'doy, un disciple du célèbre "père des moines" Mār Awgin, qui n'avait jusqu'ici fait l'objet d'aucune traduction; S. P. Brock en offre une belle version, fixant la datation du texte au VIII ${ }^{\mathrm{e}}$ siècle ("The History of Mar Yawnan », p. 1-42). Ce récit constitue un témoignage de premier ordre sur le développement de la vie monastique dans la région du golfe Persique. Pour le genre épistolaire sont présentées et traduites à nouveau frais les cinq 
missives du catholicos Ǐšōyahb III d'Adiabène à l'intention des chrétiens du BēthQațrāyē, rédigées vers 650 au moment de la crise sécessionniste du Fārs (M. Kozah, "Isho'yahb III of Adiabene's Letters to the Qataris », p. 43-88 avec texte syriaque). Ces lettres sont des documents historiques importants pour comprendre les évolutions socio-religieuses des communautés de ces espaces méridionaux au moment où se met en place une nouvelle hégémonie politique avec l'arrivée des conquérants arabes. Un renvoi à quelques-unes des nombreuses études sur le sujet aurait été nécessaire. Puis viennent la présentations de trois auteurs représentatifs mais moins connus, avec un choix de leur production: Ahūb du Qatar, dont est présenté le Livre de la finalité des Psaumes (M. Kozah, p. 89-90), avec une traduction inédite en anglais (B. ter Haar Romeny, "Introduction to the Book of the Aims of the Psalms by Ahūb Qațraya », p. 90-95); Abraham bar Lipeh et son commentaire des offices liturgiques en version anglaise avec édition du texte syriaque (M. Kozah, "Abraham Qațraya bar Lipah's Commentary on the Liturgical Offices ", p. 97-146); le petit Livre législatif de Mār Šem'ūn par un moine anonyme (M. Kozah, "Preface to Mar Shem'ūn's Law Book by an Anonymous Monk from Beth Qațraye ", p. 147-153). Les deux articles suivants ont à voir avec le célèbre Dadī̌š` Qațrāyā dont le commentaire abrégé du Paradis des Pères d'Égypte de 'Enanī̌šc‘ (sur modèle de l'œuvre de Pallade) eut un retentissement considérable dans le monachisme chrétien oriental: une traduction est effectuée à partir d'un manuscrit en garshuni (M. Kozah, A. Abu-Husayn, «Compendious Commentary on the Paradise of the Egyptian Fathers in Garshuni », p. 155-187) ; notons qu'une édition critique commentée de ce texte a été préparée chez le même éditeur : M. Kozah, A. Abu-Husayn, S. A. Mourad (eds.), Dadisho' Qatraya's Compendious Commentary on The Paradise of the Egyptian Fathers in Garshuni (Texts from Christian Late Antiquity 43), Piscataway, 2016. R. Kitchen effectue une ouverture très intéressante en soulignant la postérité de ce commentaire dans le monachisme éthiopien qui l'intégra dans son Livre des trois moines, un ensemble de plusieurs traités dont le Filekseyus en ge'ez (attribué à Philoxène de Mabboug), qui transita du syriaque via l'arabe chrétien, un manuel ascétique sous forme de questions/réponses (genre de l'erotapokriseis) qui servit durant de longs siècles à la formation des religieux d'Ethiopie; l'A. donne ici une traduction de cet important document («Introduction to Selections from the Ge'ez Filekseyus», p. 189-252).

3 Quatre contributions sont dévolues à la figure de proue du courant monastique et mystique syro-oriental du VII ${ }^{\mathrm{e}}$. Isaac de Ninive $(\dagger 700)$ : l'une effectue la traduction des Chapitres sur la connaissance, qui s'inscrivent dans le sillage des Kephalaia d'Évagre le Pontique dont Isaac se sait héritier, un genre littéraire qui connut une réelle popularité parmi les chrétiens orientaux (G. Kessel, «Isaac of Nineveh's Chapters on Knowledge », p. 253-280). La seconde réflexion a pour objet la III partie de la production littéraire d'Isaac, dont les thématiques principales restent la prière et la vie d'intimité avec Dieu, sur fond de conseils sur la vie en solitude; $\mathrm{M}$. Hansbury en réalise une remarquable traduction annotée de plus d'une centaine de pages («Isaac the Syrian: The Third Part ", p. 281-440), et propose dans le chapitre qui suit la version anglaise de deux autres discours d'Isaac, tirés de la Ve partie («Two Discourses of the Fifth Part of Isaac the Syrian's Writings ", p. 441-470). Le dernier chapitre de l'ouvrage contient l'édition du texte garshuni des homélies ascétiques d'Isaac, tirées de la IVe partie (M. Kozah, «The Fourth Part of Isaac Qatraya's Ascetical Homilies in Garshuni », p. 471-691).

Cet ouvrage souligne à lui seul la richesse culturelle du Bēth-Qațrāyē chrétien à ces époques, alors même que s'impose déjà la culture islamique, et le rôle central que joua 
le courant monastique qui se développa dans cette région dans la diffusion et l'élaboration des savoirs au moment même de ces transformations géopolitiques.

\section{AUTEUR}

\section{FLORENCE JULLIEN}

CNRS, Mondes iranien et indien, Paris 